\title{
Machen uns nur die Gene krank?
}

\author{
Hansjakob Müller \\ Prof. em. Dr. med., Medizinische Genetik, Universitätsspital Basel
}

La maladie est-elle uniquement d'origine génétique?

Le battage publicitaire autour des tests génétiques visant à dépister les risques de maladie donne l'impression que notre avenir est déterminé avant tout par nos prédispositions génétiques. Certains affirment au contraire que la médecine actuelle accorde trop d'importance à la génétique. Qui a raison? A l'aide de plusieurs exemples, nous allons tenter de mettre en évidence les nombreuses interactions entre le génome et les autres facteurs susceptibles d'avoir un impact sur notre santé, dont la grossesse / I'accouchement, le microbiome, le comportement individuel ainsi que l'environnement social et naturel.

Marktschreierische Angebote von «Gentests» für Gesundheitsrisiken erwecken den Eindruck, dass unser Schicksal vorzugsweise durch die Veranlagung bestimmt werde. Andere Stimmen behaupten, dass die Genetik die heutige Medizin zu einseitig präge. Wer hat recht? Einzelbeispiele sollen illustrieren, welch vielfältige Wechselwirkungen/Abhängigkeiten zwischen dem Genom und anderen die Gesundheit gefährdenden Risikoquellen bestehen (siehe Abb. 1).

Ein Glossar findet sich am Ende des Artikels.

\section{Quellen ungünstiger Einflüsse auf unsere Gesundheit} unter besonderer Berücksichtigung der Veranlagung

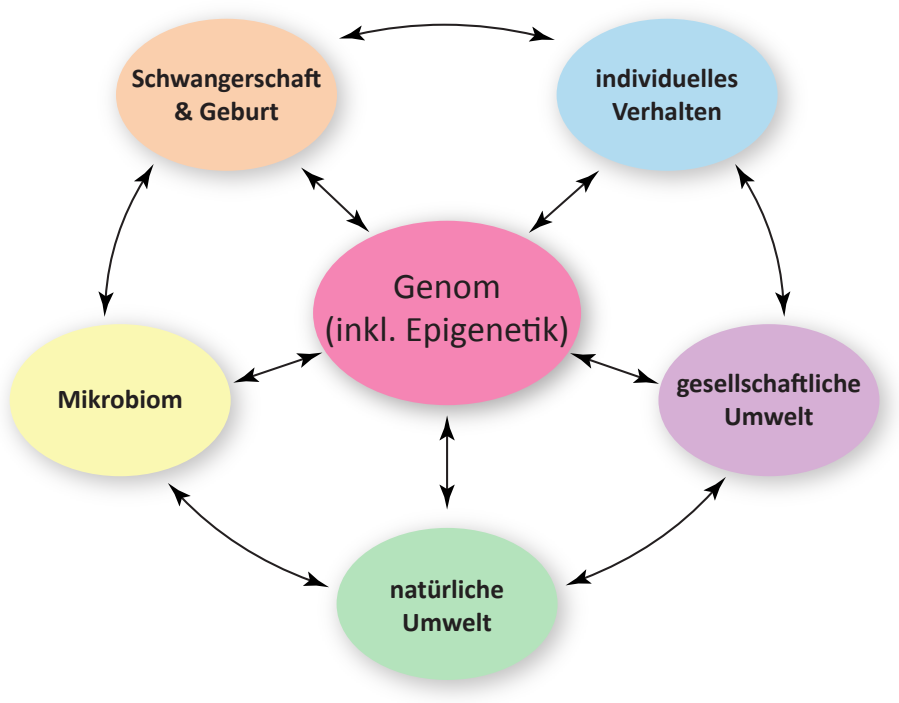

Abbildung 1: Quellen ungünstiger Einflüsse auf unsere Gesundheit.

\section{Quellen gesundheitsschädigender Einflüsse}

\section{Genom, inklusive Epigenetik}

Erbkrankheiten werden durch Mutationen der Chromosomen oder der DNA verursacht. Die durch umschriebene Chromosomen- oder Genmutationen ausgelösten Krankheitsbilder variieren zwischen den betroffenen Personen beachtlich, was auf zusätzliche Einflüsse - darunter auch weitere genetische - bei deren Ausprägung hinweist.

Die häufigen multifaktoriell verursachten Krankheiten wie Diabetes mellitus, koronare Herzerkrankungen, Bluthochdruck oder Asthma resultieren aus einem ungünstigen Wechselspiel von vielen genetischen und nicht-genetischen Faktoren. Die Patienten weisen eine variable Zahl von Genvarianten sog. «Risiko-Genen» - auf, die einzeln meist einen geringen Einfluss haben. Erst deren gehäuftes Vorkommen bei einer Person führt gemeinsam mit ungünstigen Umweltbedingungen/Lebensgewohnheiten zur Erkrankung. Die Identifizierung der bei der Verursachung dieser komplexen Krankheiten beteiligten genetischen Faktoren ist das erklärte Ziel der aktuellen Genom-weiten Assoziationsstudien (= GWAS)*

Bei den monogenen Erbkrankheiten ist die DNASequenz eines einzelnen Protein-kodierenden Gens so verändert, dass es seine Funktion(en) nicht mehr erfüllen kann. Gene werden auch durch epigenetische Modifikationen wie Methylierung der Cytosinbase oder chemische Veränderung an Histonen, respektive durch ihre Positionierung im Zellkern funktionell beeinflusst. Ihre Expression wird zudem durch die RNA-Interferenz, d.h. durch ein regulatorisches Wechselspiel mit RNAonly-Genen (ncRNAs = non-coding RNAs) gesteuert. Für die Medizin ist die Erforschung epigenetischer Phänomene wichtig, um die Verursachung und Entwicklung komplexer Krankheiten verstehen zu lernen und Medikamente dagegen $\mathrm{zu}$ entwickeln. Auch uns im Verlaufe der Schwangerschaft und später im Leben prägende Einflüsse dürfen mindestens teilweise durch epigenetische Modifikationen fixiert werden. 


\section{Schwangerschaft und Geburt}

Die Mütter bestimmen unweigerlich die Umgebung, in der sich Embryonen und Feten entwickeln, was gesundheitliche Konsequenzen haben kann. Ein Beispiel dafür ist die maternal-fetale Inkompatibilität der D-Rhesusblutgruppeneigenschaft, die zur hämolytischen Neugeborenenerkrankung führt. Die Häufigkeit von Fehlbildungen und Grosswuchs mit den entsprechenden geburtshilflichen Konsequenzen ist bei Kindern von Müttern mit Diabetes mellitus erhöht. Vom Smith-Lemli-Opitz-Syndrom, einem autosomal-rezessiv vererbten, in seiner Manifestation sehr variablen Fehlbildungssyndrom betroffene Embryonen können kein eigenes Cholesterol synthetisieren; sie sind auf exogenes der Mutter angewiesen. Der mütterliche APO-E-Genotyp beeinflusst die Cholesterol-Versorgung des Ungeborenen. Die genetischen Mitursachen (zu ähnliche / zu verschiedene Eigenschaften) der Präeklampsie, einer für Mutter und Kind gefährlichen Schwangerschaftskomplikation, erweisen sich als so vielfältig, dass darauf in Fachbüchern der Genetik kaum eingegangen wird. Erbgutunverträglichkeiten wirken sich nicht nur intrauterin, sondern auch später im Leben auf den Gesundheitszustand des Nachwuchses aus. So wurden HLA-B-Inkompatibilitäten identifiziert, die zu Schizophrenie disponieren sollen.

Die Geburt gilt als die gefährlichste Phase in unserem Leben. Zahlreiche Risikofaktoren wie vorzeitiger Blasensprung oder Wehenschwäche können lebensbedrohende Probleme schaffen. Kindliche Faktoren ihrerseits wie der Hydrozephalus, eine häufige Begleitfehlbildung verschiedener Chromosomenstörungen, behindern den Geburtsvorgang. Meistens ist diese Fehlbildung jedoch das Resultat eines ungünstigen Zusammenspiels verschiedener Risikofaktoren, mütterlicher Alkoholmissbrauch oder Toxoplasmose miteingeschlossen.

\section{Mikrobiom}

Mikroorganismen, wie sie in und auf dem Körper leben, spielen für unsere Gesundheit eine grössere Rolle, als lange bekannt war. Man bemerkte zwar, dass Kinder, die vaginal geboren wurden, eine breitere mikrobiologische Flora als diejenigen nach einem Kaiserschnitt ausweisen, was sich auf die Entwicklung und Ausreifung des Immunsystems und damit auf die Entstehung von Asthma, Lebensmittelallergien oder den Diabetes Typ I auswirkt.

Die Verfahren der molekulargenetischen Analytik wurden mittlerweile zur treibenden Kraft bei der Erforschung des Mikrobioms. Veränderungen der Mikrobioten-Flora werden als zusätzliche Risikofak- toren mit einer ganzen Reihe von multifaktoriell verursachten Krankheiten in Verbindung gebracht: entzündliche Darmerkrankungen, Übergewicht und metabolisches Syndrom, Allergien und sogar neuropsychiatrische Leiden. Das ist wenig überraschend, denn die Mikrobioten produzieren wertvolle Metabolite mit antiinflammatorischer und antioxidativer Wirkung sowie Vitamine; sie verarbeiten im Darm schwerverdauliche Stoffe und verhindern auch dessen Kolonisierung mit pathogenen Keimen.

Über einen unmittelbaren Einfluss des menschlichen Erbgutes auf die Flora der Mikrobioten oder deren Interaktionen mit anderen gesundheitsgefährdenden Einflüssen ist vorerst noch wenig bekannt. Sind Veränderungen des Mikrobioms die Ursache oder die Folge von solchen? Die Zöliakie, eine bei uns häufige entzündliche Krankheit des Dünndarms, wird durch die Einnahme von Gluten ausgelöst. Ihr klinischer Schweregrad variiert beachtlich. Betroffene weisen

Kinder, die nicht per Kaiserschnitt, sondern vaginal geboren wurden, haben eine breitere mikrobiologische Flora, was sich auf das Immunsystem auswirkt.

spezifische genetische Eigenschaften des HLA-Systems auf, nämlich die Antigene HLA-DQ2/DQ8. Jedoch lange nicht alle Träger dieser HLA-Merkmale erkranken trotz Gluten-Einnahme an Zöliakie. Neuere Forschungsergebnisse deuten auch darauf hin, dass Mikrobioten für den Übergang von der Gluten-Toleranz zu einer unerwünschten Immunantwort verantwortlich sind.

\section{Individuelles Verhalten}

Gesunder Schlaf und gut funktionierende «innere Uhren" sind eine wichtige Voraussetzung für eine gute physische und psychische Gesundheit. Schlaf beruht auf einem komplexen Netzwerk steuernder Einflüsse wie Lebensgewohnheiten, aber auch genetischer. Das diesbezügliche Wissen ist noch recht begrenzt. Die Narkolepsie und einige weitere Schlafstörungen wurden mit der vererbbaren HLA-Eigenschaft DQB1*06.02 sowie anderen Gen-Polymorphismen in Zusammenhang gebracht. Gesundheitsprobleme in verschiedenen Organen entstehen dann, wenn die Funktion von Genen, die dort die Produktion von Eiweissen im Tages-/Nacht-Rhythmus steuern, gestört ist.

«Der Apfel fällt nicht weit vom Stamm.» Diese Volksweisheit gilt besonders für die Abhängigkeit von psychotropen Substanzen mit negativen Auswirkungen auch auf die körperliche Gesundheit. Die Bedeutung der Veranlagung wird durch Zwillingsstudien und zu- 
nehmend durch GWAS gestützt. Eltern vererben ihren Kindern aber nicht nur Teile des Erbgutes; sie bestimmen auch deren näheres soziales Umfeld. Das glaubwürdig gelebte Vorbild im Umgang mit Suchtmitteln gilt immer noch als die beste Präventionsstrategie auf familiärer Ebene!

Die Alkoholabhängigkeit wird wie andere Suchterkrankungen entscheidend durch die Veranlagung mitgeprägt. Mehrere Gene und DNA-Loci wurden identifiziert, die sich auf die Persönlichkeit der betroffenen Personen, den Alkoholmetabolismus und die Entwicklung einer Alkoholtoleranz auswirken. Ein-

\section{Die Alkoholabhängigkeit wird wie andere Suchterkrankungen entscheidend durch die Veranlagung mitgeprägt.}

zelne dieser Gene scheinen für den Alkoholismus spezifisch zu sein, andere prädisponieren eher allgemein zur Abhängigkeit von psychoaktiven Substanzen. Während die Neigung zum Alkoholismus bei einzelnen Menschen genetisch begünstigt sein kann, werden andere durch ihre Veranlagung davor geschützt; sie weisen eine Alkoholintoleranz auf. Ihr AldehydDehydrogenase-2-Mangel führt bei Alkoholkonsum zum unangenehmen Acetaldehyd-Syndrom, was sie von einem Alkoholgenuss abhält.

Zwillingsstudien lassen darauf schliessen, dass auch bei der Auslösung von Verhaltenssüchten wie Kaufsucht, Spielsucht oder pathologischem Internet- oder Handy-Gebrauch genetische Faktoren beteiligt sind, ohne dass sich jeweils ein spezifisches "Sucht-Gen" identifizieren lässt. Vielmehr scheinen auch diese Verhaltensweisen multifaktorielle Ursachen zu haben.

\section{Gesellschaftliche Umwelt}

Durch uns verursachte Umwelteinflüsse, gegen die sich das einzelne Individuum kaum wehren kann, wie Verkehr und Industrie, deren Lärm und Luftverschmutzung, zu laute Musik, aber auch Antibiotika im Fleisch sowie Pestizide in Obst und Gemüse gefährden unsere Gesundheit. Vom Menschen herbeigeführte Katastrophen, Kriege, führen zu Verletzungen, Hungersnöten und verheerenden Krankheiten. Die in Europa häufige für die HFE-Hämochromatose verantwortliche Genmutation $\mathrm{C} 282$ Y zeigt ein Verteilungsmuster, das vermuten lässt, dass sie sich mit den kriegerischen Wikingern und später den Normannen verbreitete, weil sie vor einem Eisenmangel wegen Blutverlust nicht nur während der Menstruation, sondern vielleicht auch nach Verletzungen schützt.

Die Veranlagung kann sich der vom Menschen geschaffenen Umwelt anpassen. Dies zeigt die neolithi- sche Revolution. Bei den meisten Bewohnern Europas, Afrikas und des Nahen Ostens, wo man in den letzten 10000 Jahren dazu übergegangen ist, Milch von Kühen, Ziegen oder Kamelen als Nahrungsmittel auch im Erwachsenenalter zu nutzen, breiteten sich mindestens vier regional unterschiedliche Mutationen einer spezifischen Sequenz des MCM6-Gens aus, das die Expression des Laktase(LCT)-Gens steuert Konnte der Mensch zuvor die Laktose nur im Säuglingsalter verdauen, so ermöglichen diese Mutationen die Fortsetzung der Produktion des die Laktose spaltenden Enzyms im Dünndarm während des ganzen Lebens. Da dies in Viehwirtschaft betreibenden Populationen ein Überlebensvorteil war, nahm deren Trägerschaft dort rasch zu. Heute leidet in Westeuropa nur noch knapp ein Viertel der Bevölkerung an der Laktoseintoleranz.

Therapeutische Massnahmen führen zu einer $\mathrm{Zu}$ nahme von mutierten Genen in unserer Gesellschaft, indem deren Träger, die früher das reproduktionsfähige Alter nicht erreichten, sich heute fortpflanzen und ihre mutierten Gene weitergeben können. Beispiele dafür sind das Neugeborenen-Screening für die monogen verursachten Stoffwechselkrankheiten wie die Phenylketonurie mit anschliessender Diätbehandlung oder chirurgische Korrekturen der multifaktoriell verursachten Herzfehler.

Sitten und Gebräuche beeinflussen die Partnerwahl und Familienpolitik, was sich auf das Erbgut der Nachkommen auswirkt. Zeugen Cousine und Cousin ersten Grades ein gemeinsames Kind, so verdoppelt sich das Risiko, dass dieses eine schwere Behinderung aufweisen wird. Auch der IQ ist häufig vermindert. Wir alle haben einige mutierte Gene. Deren Funktionseinbusse kann jedoch - zumindest beim rezessiven Erbgang - durch die normalen auf dem anderen homologen Chromosom kompensiert werden. Bei blutsverwandten Paaren steigt die Wahrscheinlichkeit, dieselbe krankheitsverursachende Version eines Gens des gemeinsamen Vorfahrens aufzuweisen. Ihre Kinder haben dann ein Risiko von $25 \%$, dieses im homozygoten Zustand zu erben.

Schliesslich gibt es kulturelle Unterschiede in der Wahrnehmung, der Bewertung und der Behandlung von Krankheitssymptomen. Was ist eine leichte, was eine schwere Krankheit?

\section{Natürliche Umwelt}

Naturkatastrophen wie Bergstürze oder Lawinen gefährden unsere Gesundheit auch ohne menschliches Zutun. Der Klimawandel mit der Zunahme von Extremwettererscheinungen wie Hitzewellen hat gesundheitliche Auswirkungen physischer (Verletzungen, Infek- 
tionen) und psychischer Art (Stress, Angstzustände, Traumata, Depressionen). Dieser wird von uns Menschen durch den $\mathrm{CO}_{2}$-Ausstoss der Verbrennungsmotoren und -öfen, durch die Emission von Aerosolen, das Abholzen oder durch die Tierzucht begünstigt. In der Interaktion mit der natürlichen UV-Strahlung

\section{Unser Wissen über die Rolle der Veranlagung bei der Auslösung und Ausprägung vieler Krankheiten ist noch sehr begrenzt.}

im Sonnenlicht oder mit Infektionserregern hat die Veranlagung gesundheitliche Relevanz. Die dunkle Hautfarbe verhindert eine photochemische Auslösung von DNA-Schäden durch UV-Strahlen. GenMutationen, die zur Hellhäutigkeit führen, haben sich bei denjenigen Menschen verbreitet, die in nördliche Regionen auswanderten, wo weniger Sonnenlicht vorhanden ist, das zur Bildung von Vitamin D aus 7-Dehydrocholesterol benötigt wird. Bestimmte Varianten

\begin{tabular}{ll} 
Glossar & \\
\hline APO-E-Genotyp & $\begin{array}{l}\text { Erbinformation für das Apolipoprotein E, eine chemische } \\
\text { Verbindung mit wichtigen Funktionen im Fettstoffwechsel. }\end{array}$ \\
\hline Exom & $\begin{array}{l}\text { Gesamtheit aller bekannten kodierenden Abschnitte } \\
\text { des Genoms (= Exons aller Gene). }\end{array}$ \\
\hline GWAS & "genome-wide association studies»: Absuchen des gesamten \\
& $\begin{array}{l}\text { Genoms nach Nukleotidpolymorphismen (SNPs = single } \\
\text { nucleotide polymorphisms), die Risikofaktoren für multifakto- } \\
\text { riell verursachte, komplexe Krankheiten sind/sein könnten. }\end{array}$ \\
\hline Heterozygotenvorteil & $\begin{array}{l}\text { Phänomen, dass Individuen, die von einem Gen zwei verschie- } \\
\text { dene Versionen aufweisen, einen besseren Fortpflanzungs- } \\
\text { erfolg als homozygote Genträger haben. }\end{array}$ \\
\hline Histone & $\begin{array}{l}\text { Während der Evolution konservierte kleine basische Eiweiss- } \\
\text { moleküle zur Verpackung der DNA im Zellkern. }\end{array}$ \\
\hline HLA-System & $\begin{array}{l}\text { «human leucocyte antigen»-System: ausserordentlich } \\
\text { polymorphe Antigenstruktur der Leukozyten. }\end{array}$ \\
\hline Biologische Mechanismen (Chronobiologie), die die \\
verschiedenen Rythmen aufrechterhalten. Der zirkadische \\
Rhythmus wird beim Menschen vom Nucleus suprachias- \\
maticus hinter dem Nasenbein kontrolliert. Periphere zelluläre \\
"Uhren» sind in den einzelnen Organen aktiv.
\end{tabular}

des Hämoglobins oder der Glukose-6-Phosphatdehydrogenase wirken bei ihren heterozygoten Trägern als Schutzfaktoren gegen Malaria. Dies erklärt deren häufiges Vorkommen in vom Plasmodium falciparum verseuchten Regionen. Auch die Häufigkeit umschriebener Mutationen für die zystische Fibrose, die kongenitale Nebennierenrindenhyperplasie oder den Morbus Tay-Sachs lässt einen Heterozygotenvorteil gegenüber verschiedenen Infektionserregern postulieren.

\section{Abschliessende Bemerkungen}

Unser Wissen über die Rolle der Veranlagung bei der Auslösung und Ausprägung vieler Krankheiten ist noch sehr begrenzt; dies gilt selbst für den Zusammenhang zwischen der Symptomatik der monogen vererbten Krankheiten und den diese verursachenden DNA-Mutationen. Somit gibt es viel Raum für Spekulationen über die Verursachung und die Entwicklung von Krankheiten. Wir brauchen zweifelsohne mehr Forschung, d.h. Studien mit einer Vielzahl von Teilnehmerinnen und Teilnehmern, die zur Freigabe ihrer genetischen und medizinischen Daten bereit sind. Die dabei erzielten Resultate eröffnen einen verbesserten Einblick in die biologischen Prozesse, die Krankheiten zugrunde liegen, und damit auch das Ableiten neuartiger Therapien.

Krankheitsveranlagungen lassen sich nicht wegzaubern! Betroffene müssen damit leben. Sie wollen als Mitmenschen wie du und ich akzeptiert und integriert werden! Grundsätzlich sind sie ja auch nicht anders, denn wir alle haben einige ungünstige Erbfaktoren, glücklicherweise oft mit weniger nachteiligen Auswirkungen. Neben besseren naturwissenschaftlichen Kenntnissen über Krankheitsveranlagungen und den daraus ableitbaren präventiven und therapeutischen Massnahmen sind mehr Einfühlungsvermögen, Verständnis, Toleranz, Hilfsbereitschaft, Solidarität im Zusammenleben mit genetisch Behinderten gefragt.

\section{Danksagung}

Herzlichen Dank meinen Kollegen Prof. Sven Cichon und Karl Heinimann für das kritische Gegenlesen.

Weiterführende Literatur kann beim Autor erfragt werden 